\title{
A NOTE ON TRIVIAL FIBRATIONS
}

\author{
Petar PAVEŠIĆ \\ University of Ljubljana, Slovenia
}

\begin{abstract}
We study the conditions on spaces $B$ and $F$ given which, every fibration with base $B$ or with fibre $F$ is fibre-homotopy trivial. In particular, we prove that every fibration whose base is a CW-complex and fibre an Eilenberg-MacLane space $K(G, 1)$ with $G$ a complete group is fibre-homotopy trivial.
\end{abstract}

\section{INTRODUCTION}

It is well-known that every fibration over a contractible base is fibre homotopically trivial. In fact, it is easy to see that this property characterizes contractible spaces. It is less clear whether a fibration with contractible fibre must also be fibre homotopically trivial, and whether the triviality of all fibrations with a given fibre implies the contractibility of that fibre. In this paper we study complexes with a surprising property that they are noncontractible and yet they can only be fibres of trivial fibrations.

In the first section we list the relevant facts about fibrations and their classification, while in the second section we study bases and fibres that automatically make fibrations trivial.

\section{Preliminaries on fibrations}

Although the classification of fibrations is formally analogous to the classification of fibre bundles, there are some delicate issues regarding explicit constructions and the scope of the results, which is evident from a series of different approaches to these problems. To fix the ideas we give the list of definitions and results which we are going to use.

2010 Mathematics Subject Classification. 55R35.

Key words and phrases. Fibration, fibre-homotopy equivalence, complete group.

Partly supported by the Ministry for Education, Science and Sport of the Republic of Slovenia research program No. 0101-509. 
By fibration we mean a projection $p: E \rightarrow B$ which has the homotopy lifting property from arbitrary spaces (i.e., a Hurewicz fibration). If $B$ is path-connected then all fibres of $p$ are homotopy equivalent so we will often use the notation $F \hookrightarrow E \stackrel{p}{\longrightarrow} B$, where $F$ denotes the fibre over some point in $B$.

Fibrations $p: E \rightarrow B$ and $p^{\prime}: E^{\prime} \rightarrow B$ are fibre-homotopy equivalent if there are fibre-preserving maps $f: E \rightarrow E^{\prime}$ and $g: E^{\prime} \rightarrow E$ and fibrepreserving homotopies between $f g$ and $1_{E}$, and between $g f$ and $1_{E^{\prime}}$.

There is a technical difficulty when considering fibrations with respect to the relation of fibre-homotopy equivalence, because a fibre space (i.e., a fibre-wise space in the sense of [6]) which is fibre-homotopy equivalent to a fibration is not necessarily a fibration. To avoid this difficulty we define a fibre space $p: E \rightarrow B$ to be an $h$-fibration, if it is fibre-homotopy equivalent to a fibration, or equivalently if it satisfies the following weak homotopy lifting property: for every map $h: A \rightarrow E$ and every homotopy $H: A \times I \rightarrow B$ starting at $H_{0}=p h$ there is a homotopy $\widetilde{H}: A \times I \rightarrow E$ which covers $H$ but $\widetilde{H}_{0}$ is only fibre-homotopic to $h$. Clearly, the classification of fibrations up to fibre-homotopy equivalence coincides with that of $h$-fibrations.

A fibration $F \hookrightarrow E \stackrel{p}{\longrightarrow} B$ is fibre-homotopy trivial if it is fibre-homotopy equivalent to the product fibration $\mathrm{pr}_{B}: B \times F \rightarrow B$. A fibration is locally trivial if there is a covering $\left\{U_{\lambda}\right\}$ of the base $B$, such that the restrictions $p: p^{-1}\left(U_{\lambda}\right) \rightarrow U_{\lambda}$ are fibre-homotopy trivial. We usually require that the covering $\left\{U_{\lambda}\right\}$ is numerable, i.e., that admits a subordinated partition of unity. When a fibration is locally trivial with respect to a numerable covering, we say that it is numerably trivial. The existence of a partition of unity is a technical condition that allows gluing of maps and spaces, and is required in the classification theorems.

If $p: E \rightarrow B$ is a fibration, and if $U \subseteq B$ can be deformed in $B$ to a point (i.e., if the inclusion $U \hookrightarrow$ is homotopic to a constant map), then the restriction $p: p^{-1}(U) \rightarrow U$ is fibre-homotopy trivial. This fact motivates the following definition: a space $B$ which admits a numerable covering $\left\{U_{\lambda}\right\}$ such that all $U_{\lambda}$ can be deformed in $B$ to a point is called Dold space (cf. $[8,9]$ ). The class of Dold spaces contains all spaces of the homotopy type of a CWcomplex, all paracompact locally contractible spaces, the classifying spaces of topological monoids, unreduced suspensions and joins. Clearly, every fibration whose base is a Dold space is numerably trivial.

The well-known classification of fibre bundles with fibre $F$ and structure group $G$ through homotopy classes of maps into classifying spaces can be extended to fibrations. In that case the role of the structure group is taken by the space $\operatorname{aut}(F)$ of all self-homotopy equivalences of the fibre $F$. Since $\operatorname{aut}(F)$ is only a topological monoid and not a group, the construction of the corresponding classifying space is not as straightforward as in the case 
of groups, and there are several variants, each of them with its advantages and disadvantages. We are going to use the formulation of [4] that there is a universal fibration $F \hookrightarrow B$ aut $_{*}(F) \rightarrow B$ aut $(F)$ that classifies fibrations over $\mathrm{CW}$-complexes, in the sense that for every $\mathrm{CW}$-complex $B$ the pullback operation induces a bijection between $[B, B$ aut $(F)]$ and the set of fibrehomotopy equivalence classes of $(h)$-fibrations with fibre $F$ over $B$. Here $\operatorname{aut}_{*}(F)$ denotes the space of self-homotopy equivalences of $F$ which preserve the base-point, and $B(-)$ is a functor which for every topological monoid with a homotopy inverse $H$ constructs the corresponding classifying space $B H$. The functor $B$ is inverse to the loop-space construction in the sense that $B(\Omega X) \simeq X$ and the classifying fibration corresponds to the prolongation (in the sense of the Puppe-Barratt sequences) of the evaluation fibration $\operatorname{aut}_{*}(F) \hookrightarrow \operatorname{aut}(F) \stackrel{\mathrm{ev}}{\longrightarrow} F$.

\section{Automatically trivial fibrations}

Let us begin with the following characterization of the contractibility of a space $B$ in terms of fibrations over $B$. If $B$ is contractible then every $h$ fibration over $B$ is fiber-homotopy trivial. This implies in particular that the path fibration $\Omega B \hookrightarrow P B \rightarrow B$ must be trivial. As the based path space $P B$ is contractible, the homotopy equivalence $P B \simeq B \times \Omega B$ implies that $B$ is contractible. Thus we have proved the following result.

Proposition 2.1. The following conditions are equivalent for an arbitrary space $B$.

1. $B$ is contractible;

2. The path fibration over $B$ is fiber-homotopy trivial.

3. Every fibration with base $B$ is fiber-homotopy trivial.

We are going to show that the situation is more complex when one tries to relate the contractibility of the fibre with the triviality of the fibration.

Proposition 2.2. Every numerably trivial h-fibration with contractible fibre is fibre-homotopy trivial.

Proof. By the equivalence between (d) and (e) in Theorem 3.1. of [3] every such fibration is locally shrinkable, which by Corollary 3.3.of [3] implies that it is fibre-homotopy trivial.

In particular, every $h$-fibration with contractible fibre over a Dold space is fibre homotopy trivial. This is however not true for arbitrary bases, as can be seen from the following example.

EXAMPLE 2.3. Let $W$ denote the Warsaw circle: then the space of loops $\Omega W$ is contractible, which means that every numerably trivial fibration with fibre $\Omega W$ must be fibre-homotopy trivial. However, the path fibration over 
$\Omega W \hookrightarrow P W \rightarrow W$ cannot be fibre-homotopy trivial, as it would imply that $W \times \Omega W \simeq P W \simeq *$, which is impossible, since $W$ is not contractible. Therefore, the above proposition cannot be extended to fibrations which are not numerably trivial.

Is there a test fibration with fibre $F$ (like the path-fibration for base $B$ ) whose triviality would imply that all fibrations with fibre $F$ are fibre-homotopy trivial? If we restrict our attention to fibrations over $\mathrm{CW}$-complexes, then the natural candidate is the classifying fibration

$$
F \hookrightarrow B \operatorname{aut}_{*}(F) \rightarrow B \text { aut }(F),
$$

described in the previous section. Of course, since the total space $B$ aut $_{*}(F)$ is in general not contractible, we cannot deduce that the triviality of the classifying fibrations implies the contractibility of $F$ but we have the following characterization instead:

THEOREM 2.4. The following conditions are equivalent for a $C W$-complex F:

1. Every fibration with fibre $F$ over a $C W$-complex is fibre-homotopy trivial.

2. The classifying space $B$ aut $(F)$ is weakly contractible.

3. The inclusion map $F \hookrightarrow B$ aut $_{*}(F)$ is a weak homotopy equivalence. Moreover, the above conditions are implied by

4. The classifying fibration $F \hookrightarrow B \operatorname{aut}_{*}(F) \rightarrow B \operatorname{aut}(F)$ is fibrehomotopy trivial.

If $\operatorname{aut}(F)$ has the homotopy type of a $C W$-complex then 1.-4. are all equivalent.

Proof. The equivalence between 1. and 2. follows from the fact that a space is weakly contractible if, and only if every map from a CW-complex to it is nulhomotopic. The equivalence between 2. and 3. follows from the homotopy exact sequence of the classifying fibration. Condition 4. clearly implies 1., and if $\operatorname{aut}(F)$ is a CW-complex then so is $B \operatorname{aut}(F)$ so 1 . implies that $[B \operatorname{aut}(F), B$ aut $(F)]$ is trivial, hence $B$ aut $(F)$ is contractible.

It is not at all clear, whether there exists a non-contractible space $F$ satisfying the above conditions. To find an answer, let us consider the evaluation fibration $\operatorname{aut}_{*}(F) \hookrightarrow \operatorname{aut}(F) \stackrel{\mathrm{ev}}{\longrightarrow} F$, which is the continuation of the Puppe-Barratt sequence of the classifying fibration, and which therefore contains the same homotopy information.

If $F$ is a connected $H$-space, then the multiplication by an element $x \in F$ determines a translation $\tau_{x}$, which is a self-homotopy equivalence of $F$ (cf. Theorem 1.3.1. of [10]). Since we can always assume that $F$ has a strict unit, the map $F \rightarrow \operatorname{aut}(F), x \mapsto \tau_{x}$ determines the section of the evaluation fibration ev: $\operatorname{aut}(F) \rightarrow F$, therefore $\operatorname{aut}(F) \simeq F \times \operatorname{aut}_{*}(F)$. 
Proposition 2.5. Assume that a connected $C W$-complex $F$ admits an $H$-space structure. Then every fibration with fibre $F$ and base a $C W$-complex is trivial if, and only if $F$ is contractible.

In particular, the assumptions of the Propositions are satisfied when $F$ is an Eilenberg-MacLane space $K(G, n)$ with $G$ abelian.

The case $F=K(G, 1)$ with $G$ non-abelian is more complicated. We will first show that the higher homotopy groups of the space of un-based self-homotopy equivalences aut $(F)$ are trivial. Since aut $(F)$ is a topological monoid it is sufficient to prove that every $\alpha:\left(S^{n}, s_{0}\right) \rightarrow\left(\operatorname{aut}(F), 1_{F}\right)$ is freely homotopic to the constant map $c:\left(S^{n}, s_{0}\right) \rightarrow\left(\operatorname{aut}(F), 1_{F}\right)$. Let $\widehat{\alpha}: S^{n} \times F \rightarrow$ $F$ be the adjoint map of $\alpha$. Homotopy classes of maps into an EilenbergMacLane space $F=K(G, 1)$ are classified by their action on the fundamental group, which is in turn determined by the inclusion $i: F \rightarrow S^{n} \times F, u(x)=$ $\left(s_{0}, x\right)$. Since $\widehat{\alpha} u(x)=x=\widehat{c} u(x)$ (where $\widehat{c}$ is the adjoint of the constant map), we conclude that $\widehat{\alpha}$ is homotopic to $\widehat{c}$, therefore $\alpha$ is (freely) homotopic to the constant map. We conclude that aut $(F)$ is aspherical, hence $\operatorname{aut}_{*}(F)$, being the fibre of a map between two aspherical spaces, must be also aspherical.

It remains to compute $i_{\sharp}: \pi_{1}(F) \rightarrow \pi_{1}\left(B\right.$ aut $\left._{*}(F)\right)$ or equivalently $i_{\sharp}: \pi_{0}(\Omega F) \rightarrow \pi_{0}\left(\operatorname{aut}_{*}(F)\right)$, where $i: \Omega F \rightarrow \operatorname{aut}_{*}(F)$ is given by the standard action of the loops on the base to the fibre in the evaluation fibration. More explicitly, given a loop $\alpha \in \Omega(F), i(\alpha): F \rightarrow F$ is obtained by using the homotopy extension property to 'translate' the identity map on $F$ along $\alpha$. It is easily verified that $u(\alpha)_{\sharp}: \pi_{1}(F) \rightarrow \pi_{1}(F)$ is precisely the conjugation by $\alpha$, where we view $\alpha$ as an element of $\pi_{1}(F)$. If we identify $\pi_{1}(F)=\pi_{0}(\Omega F)=G$ and $\pi_{1}\left(B\right.$ aut $\left._{*}(F)\right)=\pi_{0}\left(\operatorname{aut}_{*}(F)\right)=$ Aut $G$ then $i_{\sharp}: \pi_{1}(F) \rightarrow \pi_{1}\left(B\right.$ aut $\left._{*}(F)\right)$ corresponds to the standard conjugation homomorphism $c: G \rightarrow \operatorname{Aut}(G)$, given by $[c(g)](h)=g^{-1} h g$. We conclude that $F \hookrightarrow B$ aut $_{*}(F)$ is a weak homotopy equivalence if, and only if the above homomorphism is bijective, so by Theorem 2.4 we obtain the following characterization.

THEOREM 2.6. Every fibration with fibre $K(G, 1)$ and base a $C W$-complex is fibre-homotopy trivial if, and only if the conjugation homomorphism $G \rightarrow$ $\operatorname{Aut}(G)$ is an isomorphism.

The groups for which the conjugation homomorphism is an isomorphism are called complete groups and are much studied especially in connection with simple groups. Examples of complete groups include all symmetric groups $S_{n}$ except for $n=2,6$ (see [7, Theorem 7.5]). By perusing the Atlas of Finite Groups we find that generically the simple groups are not complete. In particular, none of the alternating groups $A_{n}$ and only a few among linear groups are complete. All sporadic groups are either complete (e.g. the Mathieu groups $\mathrm{M}_{11}, \mathrm{M}_{23}, \mathrm{M}_{24}$, Conway groups $\mathrm{Co}_{1}, \mathrm{Co}_{2}, \mathrm{Co}_{3}$, Janko groups $\mathrm{J}_{1}, J_{4}$, the Baby Monster, the Monster, and few others), or have the outer automorphism group of order 2. Moreover by a famous theorem of Burnside, 
the automorphism group of every non-abelian simple group is complete (see [7, Theorem 7.14]). Another source of complete groups is Wielandt's theorem on the automorphism tower, which says that for every finite group the sequence

$$
G \rightarrow \operatorname{Aut}(G) \rightarrow \operatorname{Aut}(\operatorname{Aut}(G)) \rightarrow \ldots
$$

stabilizes after finitely many iterations, and the limit is obviously a complete group.

To complete the picture, let us mention a characterization of complete groups due to Hölder and Baer, that is an algebraic counterpart of Theorem 2.6: a group $G$ is complete if, and only if, whenever $G$ is a normal subgroup of a group $H$ it follows that $G$ is a direct factor of $H$ (see [7], Theorems 7.15 and 7.17).

Is there any other example of a fibre, which would automatically cause the triviality of the fibrations? If $F$ is a simply-connected coH-space then by [1, Proposition 1.13], $F$ has a homotopy inverse, which is clearly a homotopy equivalence of order 2. Therefore $\pi_{0}\left(\operatorname{aut}_{*}(F)\right)$ is non-trivial, while $\pi(\Omega F)=$ $\pi_{1}(F)=0$ so $\Omega F \not \operatorname{aut}_{*}(F)$.

If $F$ is simply connected and homotopically $n$-dimensional (i.e., $\pi_{n}(F) \neq 0$ and $\pi_{k}(F)=0$ for $k>n$ ) then we can use the spectral sequence, developed by G. Didierjean ([2]) (a variant of the Federer spectral sequence) that computes the homotopy groups of $\operatorname{aut}_{*}(F)$. When $F$ is as above the spectral sequence collapses and yields $\pi_{n}\left(\operatorname{aut}_{*}(F)\right)=\pi_{n}(F) \neq 0$. As $\pi_{n}(\Omega F)=\pi_{n+1}(F)=0$ it follows that $\Omega F \not$ aut $_{*}(F)$. For general $F$ the structure of the spectral sequence is much more complicated, but it still seems unplausible that for a non-contractible simply connected complex $\Omega F$ and $\operatorname{aut}_{*}(F)$ have isomorphic homotopy groups, so we are lead to the following:

Conjecture: For a connected CW-complex $F$ the loop-space $\Omega F$ is weakly homotopy equivalent to $\operatorname{aut}_{*}(F)$ if, and only if $F=K(G, 1)$ for a complete group $G$.

Finally, let us observe that since there exist non-trivial fibrations with contractible total space, then up to homotopy there are no particular limitations for a space $E$ to be the total space of a non-trivial fibration. In fact $E \simeq E \times \mathbb{R}$ and the standard covering map gives a fibration $E \times \mathbb{R} \rightarrow E \times S^{1}$ which is not fibre-homotopy trivial. In this context it is more interesting to ask which spaces can be total spaces of non-trivial fibrations with base and fibre a compact CW-complex, or which suspensions can be total spaces of non-trivial fibrations (for more information see [5]).

\section{REFERENCES}

[1] M. Arkowitz, Co-H-spaces, in: Handbook of Algebraic Topology, North-Holland Amsterdam, 1995, 1143-1173.

[2] G. Didierjean, Homotopie de l'espace des equivalences d'homotopie, Trans. AMS 330 (1992), 153-163. 
[3] A. Dold, Partitions of unity in the theory of fibrations, Annal of Math. (2) 78 (1963), $223-255$.

[4] D. Gottlieb, The total space of universal fibrations, Pacific J. Math, 46, (1973), 415417.

[5] D. Gottlieb, Fibering suspensions, Houston J. Math. 4, (1978), 49-65.

[6] I. James, Fibrewise topology, Cambridge Tracts in Mathematics 91, Cambridge University Press, Cambridge, 1989.

[7] J. Rotman, An introduction to the theory of groups, Graduate Texts in Mathematics 148, Springer-Verlag, New York, 1995.

[8] E. Schwamberger and R. Vogt, Dold spaces in homotopy theory, Algebr. Geom. Topol. 9 (2009), 1585-1622.

[9] J. Smrekar, CW-type of inverse limits and function spaces, arXiv:0708.2838v1.

[10] A. Zabrodsky, Hopf spaces, North-Holland, Amsterdam, 1976.

P. Pavešić

Fakulteta za Matematiko in Fiziko

Univerza v Ljubljani

Jadranska 19, 1111 Ljubljana

Slovenija

E-mail: petar.pavesic@fmf.uni-lj.si

Received: 6.7.2010.

Revised: 28.7.2010. 